\title{
Selective Monitoring of Rutin and Quercetin based on a Novel Multi-wall Carbon Nanotube-coated Glassy Carbon Electrode Modified with Microbial Carbohydrates $\alpha$-Cyclosophorohexadecaose and Succinoglycan Monomer M3
}

\author{
Joon-Hyung Jin, Eunae Cho, Chanho Kwon, and Seunho Jung* \\ Department of Bioscience and Biotechnology, Bio/Molecular Informatics Center, Konkuk University, Seoul 143-701, Korea \\ *E-mail: shjung@konkuk.ac.kr \\ Received March 23, 2010, Accepted May 4, 2010
}

\begin{abstract}
Multi-wall carbon nanotube (MWNT)-modified glassy carbon electrodes (GCE) were prepared for simultaneous determination of rutin and quercetin. Microbial carbohydrates, $\alpha$-cyclosophorohexadecaose $(\alpha-\mathrm{C} 16)$ and succinoglycan monomer M3 (M3) were doped into MWNTs to prepare a $\alpha$-C16-doped MWNT-modified GCE $((\alpha-C 16+\mathrm{MWNTs}) /$ GCE) and a M3-doped MWNT-modified GCE ((M3 + MWNTs)/GCE), respectively. The sensitivities of the $(\alpha-\mathrm{C} 16+$ MWNTs $/$ GCE to rutin and quercetin were $34.7 \mu \mathrm{A} \cdot \mu \mathrm{M}^{-1} \cdot \mathrm{cm}^{-2}$ and $18.3 \mu \mathrm{A} \cdot \mu \mathrm{M}^{-1} \cdot \mathrm{cm}^{-2}$, respectively, in a linear range of $2 \sim 8 \mu \mathrm{M}$ at $\mathrm{pH}$ 7.2. The sensitivities of the (M3 $+\mathrm{MWNTs}) / \mathrm{GCE}$ was $2.44 \mu \mathrm{A} \cdot \mu \mathrm{M}^{-1} \cdot \mathrm{cm}^{-2}$ for rutin and 7.19 $\mu \mathrm{A} \cdot \mu \mathrm{M}^{-1} \cdot \mathrm{cm}^{-2}$ for quercetin without interference.
\end{abstract}

Key Words: $\alpha$-Cyclosophorohexadecaose, Quercetin, Rutin, Square wave voltammetry, Succinoglycan monomer

\section{Introduction}

Flavonoids have various pharmacological and therapeutic activities including the potential to make difficult-to-detect biomolecules more easily detectable. ${ }^{1-5}$ Conventional labor-intensive detection methods for flavonoids based on chromatography and spectroscopy have been recently replaced with more comprehensive electrochemical methods. ${ }^{6,7}$ However, flavonoids abundant in a variety of fruits and vegetables are chemically much less soluble in water than in organic solvents. Direct electrochemical detection of flavonoids, hence, requires modification of the sensing electrode. A strong adsorption interaction between carbon nanotubes (CNTs) and a glassy carbon electrode (GCE) provides enhanced sensitivity and excellent chemical and electrical properties to CNT-modified GCE. The CNTs-adsorbed GCE surface also blocks additional adsorption of electricalinactive byproducts of flavonoids. ${ }^{8}$ Recent works have reported electrochemical selective monitoring of some flavonoids via the separation of the peak potentials $\left(\mathrm{E}_{\mathrm{p}}\right)$ of individual flavonoid constituents in a mixture.

In this work, two different carbohydrate-modified GCEs are introduced for simultaneous determination of rutin and quercetin which cannot be accomplished with the use of a conventional $\beta$-cyclodextrin $(\beta$-CD)-doped multi-wall carbon nanotube (MWNT) film. The microbial carbohydrates $\alpha$-cyclosophorohexadecaose ( $\alpha-\mathrm{C} 16)$ from $X$. oryzae and succinoglycan monomer M3 (M3) from R. meliloti were used for modifying GCEs through a medium of MWNTs and resulted in a $\alpha$-C16-doped MWNT-modified GCE $((\alpha-\mathrm{C} 16+\mathrm{MWNTs}) / \mathrm{GCE})$ and a M3dopped MWNT-modified GCE ((M3 + MWNTs)/GCE). Complexation capability due to the large cavity of $\alpha-\mathrm{C} 16$ can provide $\alpha$-C16-doped MWNTs with a better response to the relatively large molecules of rutin. A MWNT-modified GCE (MWNTs/ GCE) was also prepared in order to clarify the electrochemical activities of $\alpha-C 16$ and M3. The sensing performance of the
$(\alpha-\mathrm{C} 16+\mathrm{MWNTs}) / \mathrm{GCE}$ and the (M3+MWNTs)/GCE were assessed by cyclic voltammetry $(\mathrm{CV})$ and square wave voltammetry (SWV) in terms of sensitivity and selectivity in a sodium phosphate buffer (SPB) solution at $\mathrm{pH} 7.2$ by comparing the peak current density $\left(\mathrm{J}_{\mathrm{p}}\right)$ and the $\mathrm{E}_{\mathrm{p}}$ arising from the redox reactions of rutin and quercetin in a potential range of $0 \sim 0.5 \mathrm{~V} v s . \mathrm{Ag} / \mathrm{AgCl}$.

\section{Materials and Methods}

Reagents and materials. M3 is an octasaccharide, having one acetyl group, one pyruvyl group, and two succinyl groups as substituents, derived from R. meliloti 1021. The oligosaccharide $\alpha-\mathrm{C} 16$ is a ring-shaped hexadecasaccharide isolated from Xanthomonas oryzae. The molecular structures of $\mathrm{M} 3$ and $\alpha-\mathrm{C} 16$ are shown in Figs. 1 (a) and (b), respectively. Figs. 1 (c) and (d) show the molecular structures of quercetin and rutin, respectively. MWNTs dissolved in ethanol (SolCNT \#3011, Cluster Instruments Co., Ltd., $1 \mathrm{wt} \%$ ) were used as an entrapping matrix for $\alpha-\mathrm{C} 16$ and M3. Rutin trihydrate $(>90 \%)$ quercetin $(>98 \%)$, $\mathrm{SPB}$ ( $\mathrm{pH} 7.2)$, and ethanol (>99.5) were used without further purification.

Equipment. Electrochemical measurements were performed in an electrochemical workstation composed of a potentiostat/ galvanostat (VersaSTAT3), an IBM-compatible PC, a lab-made electrochemical cell, and a Faraday cage. Pt wire ( $>99.99 \%)$ was used as the counter electrode. The working electrode potential was always recorded versus the $\mathrm{Ag} / \mathrm{AgCl}$ (Sodium saturated) reference electrode.

Preparation and identification of $\boldsymbol{\alpha - C 1 6}$ and M3. $X$. oryzae from the Korean Agricultural Culture Collection (KACC) was grown in a triptone glucose yeast (TGY) medium. After extraction with $5 \%$ trichloroacetic acid and chromatographic purification, $\alpha-\mathrm{C} 16$ was identified using Fourier transform nuclear magnetic resonance spectroscopy (FT-NMR) and matrix assisted laser desorption/ionization time of flight mass spectroscopy 


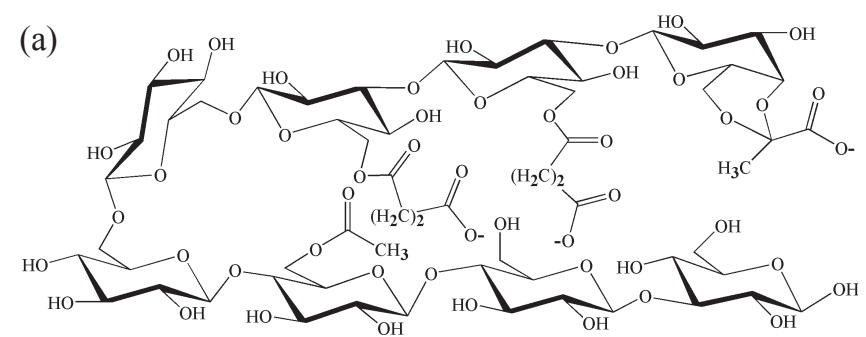

(b)

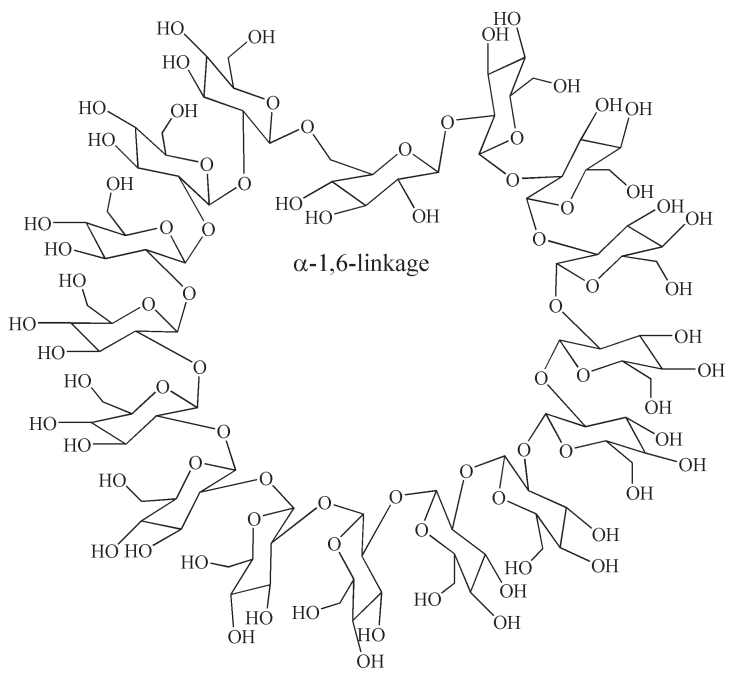

(c)

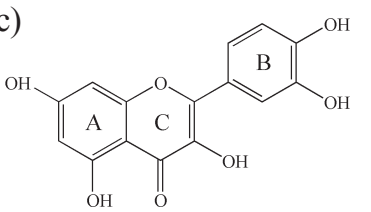

(d)

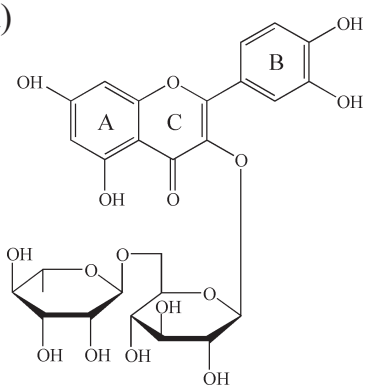

Figure 1. Molecular structure of succinoglycan monomer M3 (a), $\alpha$ cyclosophorohexadecaose (b), quercetin (c), and rutin (d). Succinoglycan monomer M3 is an open-structured octasaccharide featuring two succinyl substituents, while $\alpha$-cyclosophorohexadecaose has a closed ring structure composed of fifteen 1, 2 linkages and one alpha-1, 6 glucosidic linkage.

(MALDI-TOF). ${ }^{10,11}$ R. meliloti 1021 was cultured in a glutamate mannitol salts (GMS) medium at $30^{\circ} \mathrm{C}$ for 5 days. After multiple concentrations and centrifugations of the culture supernatants, M3 was separated from other succinoglycan monomers and analyzed by $500 \mathrm{MHz}$ NMR spectroscopy. ${ }^{12,13}$

Preparation of modified GCEs. The GCE $(3.0 \mathrm{~mm}$ in diameter, Tokai Carbon, Japan) was polished with 0.3 micron alumina powder on a polishing cloth after each use to remove adsorbed impurities. After rinsing the surface thoroughly with deionized water (D.I. water), the polished GCE was sonicated for 5 minutes to remove trace amounts of alumina powder from the surface and rinsed again with D.I. water. The electrode tip was then dipped into a $30 \%$ nitric acid solution for 20 minutes, followed by the electrode surface being scanned twenty times in a $0.5 \mathrm{M}$ (a)

(b)

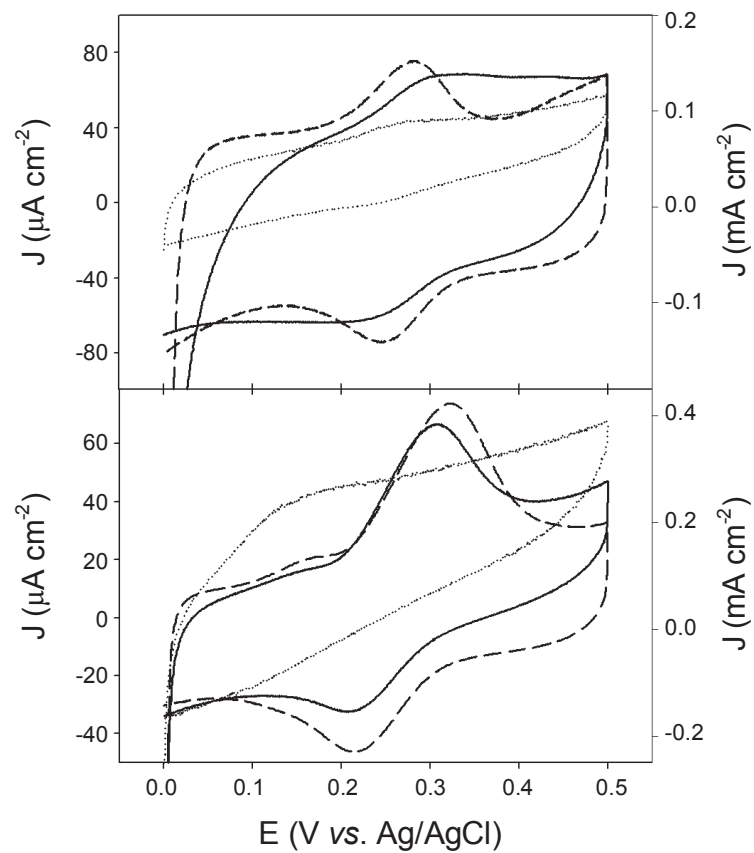

(c)

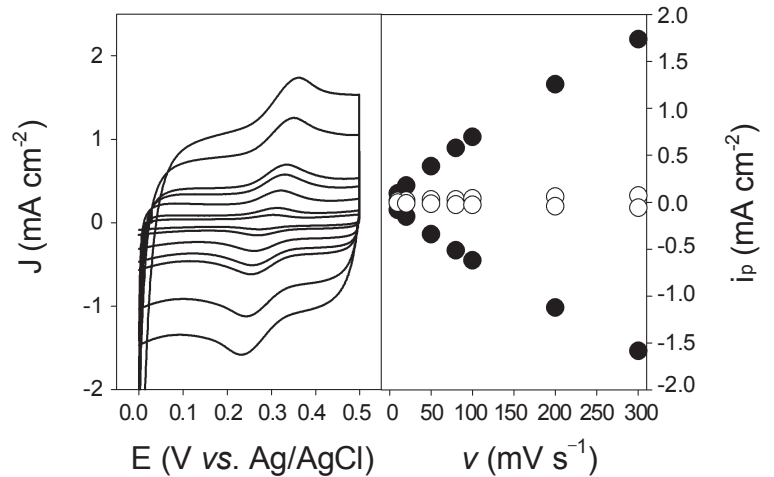

Figure 2. Cyclic voltammograms of the multi-wall carbon nanotubemodified glassy carbon electrode (solid line), the $\alpha$-cyclosophorohexadecaose-doped multi-wall carbon nanotube-modified glassy carbon electrode $((\alpha-\mathrm{C} 16+\mathrm{MWNTs}) / \mathrm{GCE})$, shown by a dashed line, and the succinoglycan monomer M3-doped multi-wall carbon nanotubemodified glassy carbon electrode ((M3 + MWNTs)/GCE), shown as a dotted line, either in the presence of $7.5 \mu \mathrm{M}$ rutin (a) or $7.5 \mu \mathrm{M}$ quercetin (b). Electrolyte $=0.15 \mathrm{M}$ sodium phosphate buffer $(\mathrm{SPB})$ solution ( $\mathrm{pH} 7.2) ; v=0.02 \mathrm{~V} \cdot \mathrm{s}^{-1}$. Note that the right axes in (a) and (b) are only for the $(\alpha-C 16+$ MWNTs $) /$ GCE. (c) shows CV diagrams of the $(\alpha-\mathrm{C} 16+\mathrm{MWNTs}) / \mathrm{GCE}$ in a SPB solution ( $\mathrm{pH}$ 7.2) containing $7.5 \mu \mathrm{M}$ rutin at $0.01 \mathrm{~V} \cdot \mathrm{s}^{-1}, 0.02 \mathrm{~V} \cdot \mathrm{s}^{-1}, 0.05 \mathrm{~V} \cdot \mathrm{s}^{-1}, 0.08 \mathrm{~V} \cdot \mathrm{s}^{-1}, 0.1 \mathrm{~V} \cdot \mathrm{s}^{-\mathrm{P}}$, $0.2 \mathrm{~V} \cdot \mathrm{s}^{-1}$ and $0.3 \mathrm{~V} \cdot \mathrm{s}^{-1}$, and the plots of $\mathrm{i}_{\mathrm{p}} v s . v$ for the $(\alpha-\mathrm{C} 16+$ MWNTs)/GCE (closed circle) and for the (M3 + MWNTs)/GCE (open circle) are also shown.

sulfuric acid solution in a range of $-0.2 \sim 1.2 \mathrm{~V}$ at $0.05 \mathrm{~V} \cdot \mathrm{s}^{-1}$.

Solution mixtures composed of carbohydrates and MWNTs for coating the electrode substrates were prepared as follows. An ethanolic MWNT solution at 1\% (w/w) concentration was diluted in D.I. water to a concentration of $2 \mathrm{mg} \cdot \mathrm{mL}^{-1}$. Ten milligrams each of $\alpha-\mathrm{C} 16$ and $\mathrm{M} 3$ were separately dissolved in $0.5 \mathrm{~mL}$ portions of the dilutions. After vortexing during mixing, $10 \mu \mathrm{L}$ portions of each solution mixture were dropped on the cleaned GCE surface and dried in a vacuum desiccator for 24 hours to 
(a)

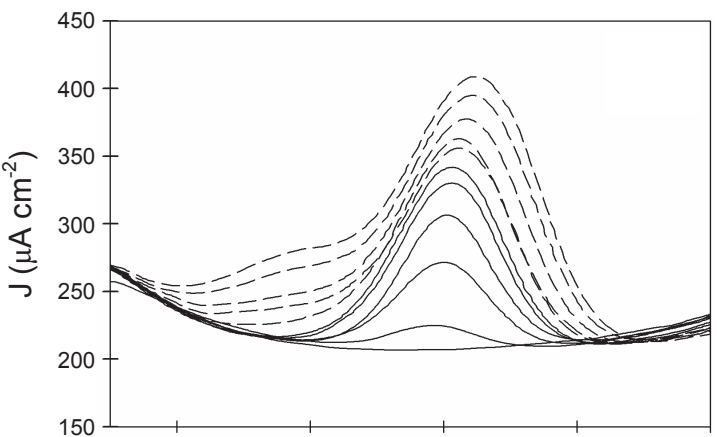

(b)

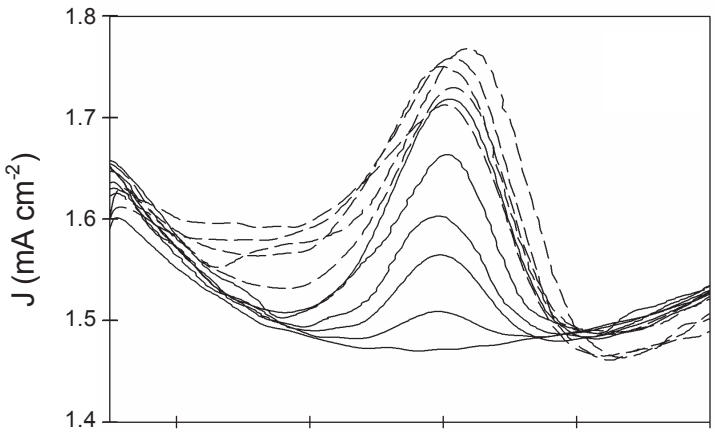

(c)

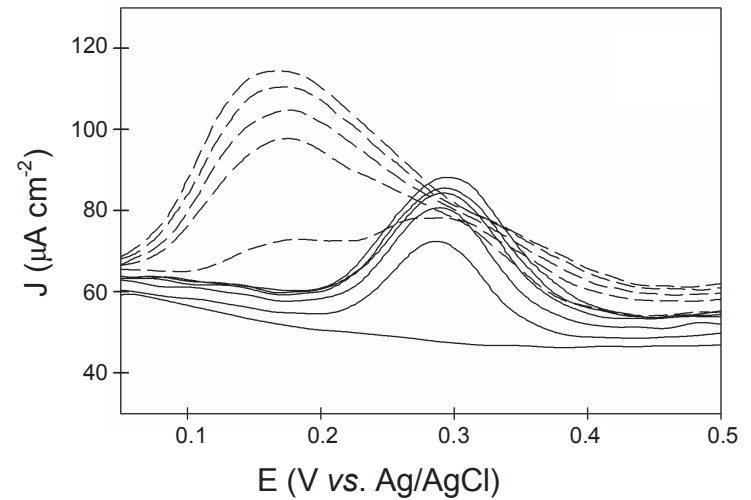

Figure 3. Square wave voltammograms of the multi-wall carbon nanotube-modified glassy carbon electrode (a), the $\alpha$-cyclosophorohexadecaose-doped multi-wall carbon nanotube-modified glassy carbon electrode (b), and the succinoglycan monomer M3-doped multi-wall carbon nanotube-modified glassy carbon electrode (c) for the oxidation of rutin and quercetin in a $0.15 \mathrm{M}$ sodium phosphate buffer solution at $\mathrm{pH}$ 7.2. Amplitude $=0.025 \mathrm{~V}$; step potential $=0.002 \mathrm{~V}$; frequency $=15 \mathrm{~Hz}$. A $10 \mu \mathrm{L}$ portion of rutin solution $(0.1 \%$ in ethanol) was added to $10 \mathrm{~mL}$ of electrolyte successively five times to obtain solid lines. $10 \mu \mathrm{L}$ of quercetin solution $(0.1 \%$ in ethanol) was then injected to the rutin-dispersed electrolyte solution five times to obtain dashed lines.

prepare the $(\alpha-\mathrm{C} 16+\mathrm{MWNTs}) / \mathrm{GCE}$, the $(\mathrm{M} 3+\mathrm{MWNTs}) / \mathrm{GCE}$, and the MWNTs/GCE.

\section{Results and Discussion}

Electrode reactions of rutin and quercetin on modified GCEs in a SPB solution at $\mathbf{p H}$ 7.2. The $\mathrm{CV}$ diagrams of rutin $(7.5 \mu \mathrm{M})$ on modified-GCEs shown in Fig. 2 (a) indicate that the redox reaction of rutin is electrochemically reversible and generates only a single oxidative peak at about $0.28 \mathrm{~V}$. Corresponding reduction peaks are observed at $0.25 \mathrm{~V}$ and the $\mathrm{E}_{\mathrm{p}}$ separation is (a)

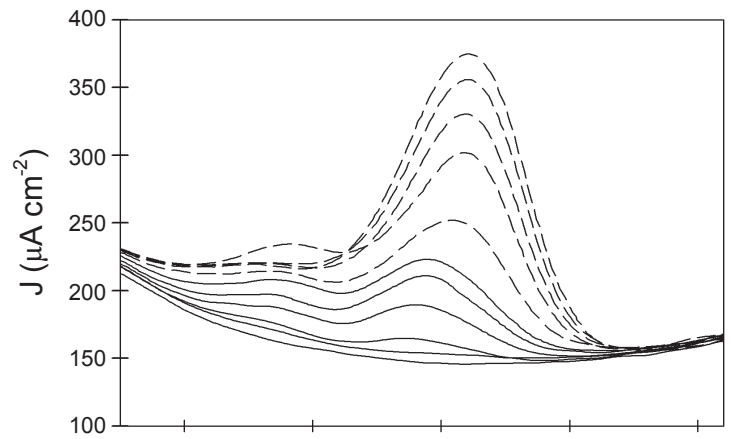

(b)

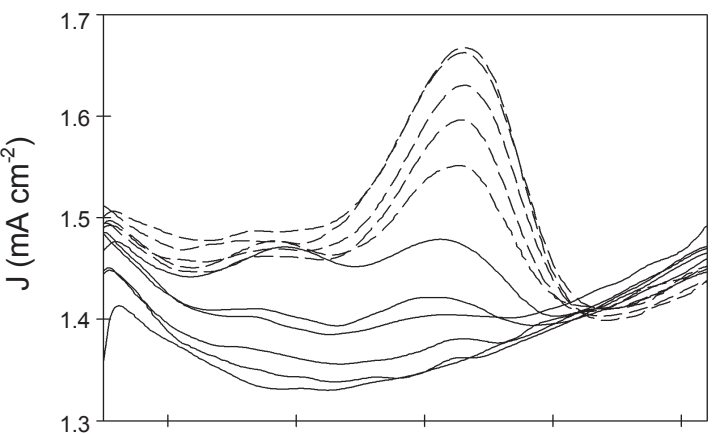

(c)

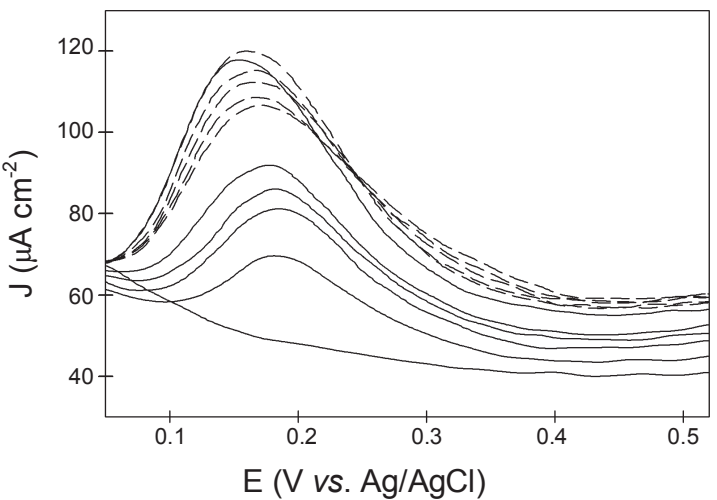

Figure 4. Square wave voltammograms of the multi-wall carbon nanotube-modified glassy carbon electrode (a), the $\alpha$-cyclosophorohexadecaose-doped multi-wall carbon nanotube-modified glassy carbon electrode (b), and the succinoglycan monomer M3-doped multi-wall carbon nanotube-modified glassy carbon electrode (c) for quercetin and rutin oxidation in a $0.15 \mathrm{M}$ sodium phosphate buffer solution at $\mathrm{pH}$ 7.2. Amplitude $=0.025 \mathrm{~V}$; step potential $=0.002 \mathrm{~V}$; frequency $=$ $15 \mathrm{~Hz}$. A $10 \mu \mathrm{L}$ portion of quercetin solution $(0.1 \%$ in ethanol) was added to $10 \mathrm{~mL}$ of electrolyte successively five times to obtain solid lines. A $10 \mu \mathrm{L}$ portion of rutin solution $(0.1 \%$ in ethanol) was then injected to the quercetin-dispersed electrolyte solution five times to obtain dashed lines.

$30 \mathrm{mV}$. The redox peak of rutin below $0.5 \mathrm{~V}$ is due to the oxidation of $3^{\prime}, 4^{\prime}$-dihydroxy groups on the ring-B of rutin and the corresponding reduction of $3^{\prime}, 4^{\prime}$-diquinone. ${ }^{14}$ Fig. 2 (b) shows $\mathrm{CV}$ diagrams of the redox reaction of quercetin $(7.5 \mu \mathrm{M})$. The MWNTs/GCE and the $(\alpha-\mathrm{C} 16+$ MWNTs $) /$ GCE show two separate oxidative peaks at $0.15 \mathrm{~V}$ and $0.31 \mathrm{~V}$, respectively. The (M3 + MWNTs)/GCE shows a single irreversible oxidation peak around 0.15 V. Fig. 2 (c) shows CV diagrams of the $(\alpha-C 16+$ $\mathrm{MWNTs}$ )/GCE at various scan rates at $7.5 \mu \mathrm{M}$ of rutin. The plots of peak current $\left(\mathrm{i}_{\mathrm{p}}\right) v \mathrm{~s}$. scan rate $(v)$ of both the $(\alpha-\mathrm{C} 16+$ MWNTs)/GCE and the (M3 + MWNTs)/GCE were linear in a 
Table 1. Summary of sensitivities taken from the square wave voltammograms of carbohydrate-doped multi-wall carbon nanotube-modified glassy carbon electrodes

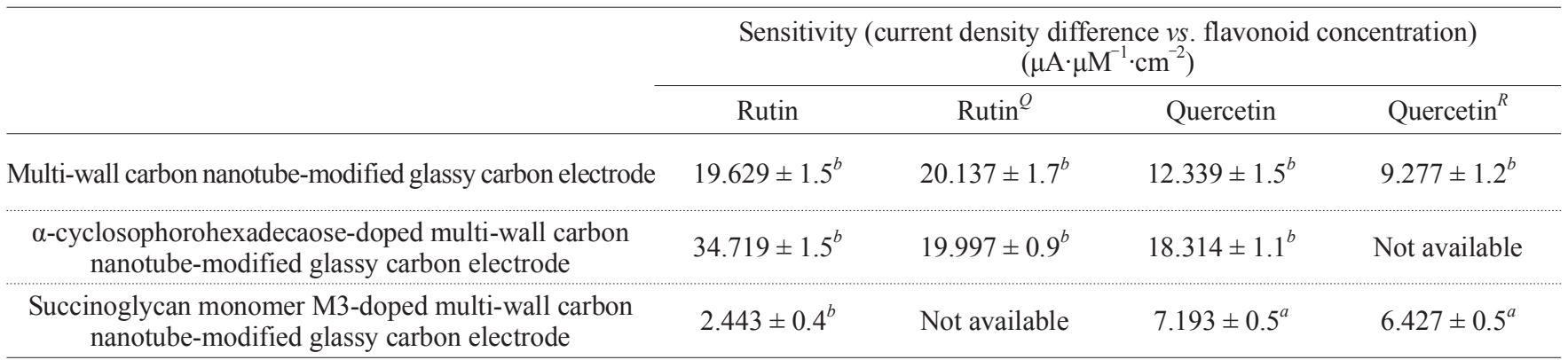

${ }^{a}$ Peak currents shown at $0.17 \mathrm{~V} v s$. $\mathrm{Ag} / \mathrm{AgCl}$ was used to calculate the sensitivity slope. ${ }^{b}$ Peak currents shown at $0.3 \mathrm{~V} v s$. $\mathrm{Ag} / \mathrm{AgCl}$ was used to calculate the sensitivity slope. ${ }^{Q}$ sensitivity to rutin in the presence of $7.5 \mu \mathrm{M}$ quercetin dispersed in an electrolyte solution. ${ }^{R}$ sensitivity to quercetin in the presence of $7.5 \mu \mathrm{M}$ rutin dispersed in an electrolyte solution.

range of $0.01 \sim 0.3 \mathrm{~V} \cdot \mathrm{s}^{-1}$. The emerging linear relationship suggests that target flavonoids are successfully captured by the carbohydrate-modified MWNT film.

Square wave voltammetric characterization of modified GCEs. The first peak group shown around $0.17 \mathrm{~V}$ is due to the oxidation of quercetin and the other peak group observed at $0.3 \mathrm{~V}$ is due to the rutin oxidation. The $\mathrm{i}_{\mathrm{p}}$ of rutin is $\mathrm{pH}$ dependent and commonly observed at $0.3 \mathrm{~V}$ under neutral conditions. ${ }^{15}$ Fig. 3 (a) shows the SWV diagrams of the MWNTs/GCE for rutin oxidation (solid lines) and for quercetin oxidation in the presence of $7.5 \mu \mathrm{M}$ rutin (dashed lines). The rutin peaks continue growing not only with increasing rutin concentration but also as quercetin concentration increases in the presence of rutin. The rutin peak of the $(\alpha-\mathrm{C} 16+$ MWNTs $) / \mathrm{GCE}$, which is 1.5 times higher than that of the MWNTs/GCE, as shown in Fig. 3 (b), is not affected by quercetin. The peak potentials of rutin and quercetin on the (M3 + MWNTs)/GCE were clearly observed at $0.28 \mathrm{~V}$ and $0.17 \mathrm{~V}$, respectively (Fig. 3 (c)). Five minutes of preconcentration before potential scanning was allowed for normalization. ${ }^{9}$ Because the redox reaction of rutin is electrochemically reversible (Fig. 2 (a)), the $\mathrm{E}_{\mathrm{p}}$ of SWV diagrams taken from the rutin oxidation approximates the formal potential of rutin. The $\mathrm{E}_{\mathrm{p}}$ of rutin varies linearly between $0.56 \mathrm{~V} \sim-0.02 \mathrm{~V}$ in a range of $\mathrm{pH} 2 \sim \mathrm{pH} 11 .{ }^{16}$ Hence, the estimated $\mathrm{E}_{\mathrm{p}}$ of rutin at $\mathrm{pH} 7.2$ given by linear interpolation $((9 \times 0.56 \mathrm{~V}-0.58 \mathrm{~V} \times$ $5.2) / 9$ ) is $0.22 \mathrm{~V}$. The roughly $60 \mathrm{mV}$ difference from the experimental value may be due to M3 molecules doped into MWNTs.

Solid lines shown in Figs. 4 (a), (b), and (c) denote SWV curves of pure quercetin at various concentrations, while all dashed lines are curves of rutin in the presence of quercetin $(7.5 \mu \mathrm{M})$. The MWNTs/GCE shows two oxidation peaks for quercetin and only one peak for rutin. The first small oxidation peak shown at $0.17 \mathrm{~V}$ is quercetin-specific and the second at $0.3 \mathrm{~V}$ is a response to both the quercetin and the rutin. The $\alpha-$ C16-doped MWNT film enhanced SWV responses to quercetin and rutin. Indeed, Fig. 3 (b) and Fig. 4 (b) indicate that the binding strength between $\alpha-\mathrm{C} 16$ and rutin is stronger than that between $\alpha-\mathrm{C} 16$ and quercetin. $\alpha-\mathrm{C} 16$, having sixteen glucose rings, appears to fit the rutin molecule better than it fits the small quercetin molecule. The single broad oxidation peak of quercetin on the (M3 + MWNTs)/GCE at $0.17 \mathrm{~V}$ is strong, as shown in Fig. 4 (c), and overspreads the region where the potential rutin- specific peak would appear. Greater sensitivity to quercetin for the M3-doped MWNT film arises from tight interaction between succinyl substituents in M3 and quercetin molecules. ${ }^{14}$

The peak current density $\left(\mathrm{J}_{\mathrm{p}}\right)$ of the rutin-specific peak shown at $0.3 \mathrm{~V}$ was used to draw calibration curves. In the case of the (M3 + MWNTs)/GCE, the quercetin-specific peak shown at $0.17 \mathrm{~V}$ was used instead. Table 1 summarizes the responses to flavonoids of all modified electrodes investigated in this work. The current density difference $(\Delta \mathrm{J})$ signifies that each $\mathrm{J}_{\mathrm{p}}$ is normalized by means of subtracting the background current density at a potential from the $\mathrm{J}_{\mathrm{P}}$ at the same potential.

\section{Conclusions}

Novel microorganism-originated carbohydrates, $\alpha-\mathrm{C} 16$ and M3, were successfully extracted, purified and immobilized on a GCE surface by using MWNTs as a conductive matrix. Although, preaccumulated flavonoid showed a screening effect, the $\alpha-\mathrm{C} 16$ present in the MWNT film as a dopant appeared to render the $(\alpha-\mathrm{C} 16+$ MWNTs $)$ composite more sensitive to both flavonoids. The structural advantage of $\alpha-\mathrm{C} 16$ for binding to rutin seems to enhance its sensitivity. The (M3 + MWNTs)/ GCE clearly separates the oxidative peak potentials of rutin and quercetin. Some functional microbial carbohydrates, which could be utilized as key components of a sensing electrode, would enlarge the potential availability of microbial carbohydrates in food and medical biosensor applications.

Acknowledgments. This work was supported by Priority Research Centers Program through the National Research Foundation of Korea (NRF) funded by the Ministry of Education, Science and Technology (2009-0093824) and partly by Basic Science Research Program through NRF funded by the Ministry of Education, Science and Technology (2009-0059986).SDG

\section{References}

1. Scalbert, A.; Manach, C.; Morand, C.; Remesy, C.; Jimenez, L. M. J. CRC Crit. Rev. Food Sci. Nutz. 2005, 45, 287.

2. Verma, A. K.; Johnson, J. A.; Gould, M. N.; Tanner, M. A. Cancer Res. 1988, 48, 5754.

3. Bao, X.; Zhu, Z.; Li, N.-Q.; Chen, J. Talanta 2001, 54, 591.

4. Kim, H.; Jeong, K.; Jung, S. Bull. Korean Chem. Soc. 2006, $27,325$. 
5. Xu, G.-R.; In, M. Y.; Yuan, Y.; Lee, J.-J.; Kim, S. Bull. Korean Chem. Soc. 2007, 28, 889.

6. Vestergaard, M.; Kerman, K.; Tamiya, E. Anal. Chim. Acta 2005 $538,273$.

7. He, J.-B.; Lin, X.-Q.; Pan, J. Electroanalysis 2005, 17, 1681.

8. Zhou, A.; Kikandi, S.; Sadik, O. A. Electrochem. Comm. 2007, 9, 2246.

9. Lin, X.-Q.; He, J.-B.; Zha, Z.-G. Sens. Actuator. B 2006, 119, 608.

10. Miller, K. J.; Kennedy, E. P.; Reinhold, V. N. Science 1986, 231, 48.

11. Vonjnov, A. A.; Slater, H.; Newman, M.; Daniels, M. J.; Dow, J.
M. Arch. Microbiol. 2001, 176, 415.

12. Wang, L. X.; Wang, Y.; Pellock, B.; Walker, G. C. J. Bacteriol. 1999, 181,6788

13. Lee, S.; Cho, E.; Kwon, C.; Jung, S. Carbohyd. Res. 2007, 342, 2682.

14. Ghica, M.-E.; Oliveira-Brett, A. M. Electroanalysis 2005, 17, 313.

15. He, J.-B.; Wang, Y.; Deng, N.; Lin, X.-Q. Bioelectrochem. 2007, $71,157$.

16. Namazian, M.; Zare, H. R.; Coote, M. L. Biophys. Chem. 2008, 132,64 . 